\title{
Reversible corpus callosum lesion in legionnaires' disease
}

\author{
J C Morgan, R Cavaliere, V C Juel
}

J Neurol Neurosurg Psychiatry 2004;75:651-654. doi: 10.1136/jnnp.2003.015669

Legionnaires' disease is often associated with neurological findings. Despite such findings, computed tomography and neuropathological investigations are typically normal. This report describes a reversible lesion of the corpus callosum identified on magnetic resonance imaging (MRI) in a patient with legionnaires' disease. MRI may show previously undocumented neuropathology in acute legionnaires' disease. Legionella pneumophila infection should be included in the differential diagnosis of conditions associated with reversible lesions of the corpus callosum.

$\mathrm{F}$ orty to fifty per cent of patients with legionnaires' disease develop neurological signs and symptoms. ${ }^{12}$ Despite frequent neurological findings in patients with this condition, neuroimaging ${ }^{13}$ and neuropathological ${ }^{3}$ studies are typically normal. We report a patient who developed neurological signs and symptoms in the setting of acute legionnaires' disease. Brain magnetic resonance imaging (MRI) showed a transient lesion in the splenium of the corpus callosum (SCC) that resolved in parallel with clinical improvement.

\section{CASE REPORT}

A middle aged man suffered three days of fever, malaise, and generalised weakness. He was subsequently admitted to the hospital after he was found unable to stand without assistance. His past medical history was unremarkable and he was not on any drug treatment. He drank two to three cans of beer a week and used no illicit drugs. On initial examination he was febrile, with moderate respiratory distress and left lower lobe rales. He was awake, oriented, and dysarthric and he had word finding difficulties. Extraocular movements were normal. He had bilateral intention tremor and dysmetria on finger-nose testing.

Laboratory abnormalities included: sodium $133 \mathrm{mmol} / \mathrm{l}$ (normal, 135-145), creatinine $141.4 \mu \mathrm{mol} / \mathrm{l}$ (61.9-114.9), glucose $16.6 \mathrm{mmol} / \mathrm{l}(3.9-5.8)$, white blood cell count $12300 /$ $\mu \mathrm{l}(4000-11000 / \mu \mathrm{l})$, platelet count $87000 / \mu \mathrm{l}$ (150 000$450000 / \mu \mathrm{l})$, alanine aminotransferase $126 \mathrm{U} / \mathrm{l}$ (7-52), aspartate aminotransferase $170 \mathrm{U} / \mathrm{l}$ (13-39), total bilirubin 70.1 $\mu \mathrm{mol} / \mathrm{l}$ (5.1-17.1), conjugated bilirubin $30.8 \mu \mathrm{mol} / \mathrm{l}(0.0-5.1)$, prothrombin time $16.8 \mathrm{~s}$ (12.5-14.6), international normalised ratio 1.3, and partial thromboplastin time $41.5 \mathrm{~s} \mathrm{(26.2-}$ 37.5). His blood ammonia level was normal at $27 \mu \mathrm{mol} / \mathrm{l}$ (9-33), and his blood ethanol level was $<10 \mathrm{mg} / \mathrm{dl}$ (repeated). Vitamin B-12, folate, thyroid stimulating hormone, hepatitis A, B, C testing, HIV 1 and 2, rapid plasma reagin, antinuclear antibody, cytomegalovirus antibody, and rickettsial antibody screens were either normal or negative. A chest $x$ ray showed left lower lobe consolidation, and an abdominal ultrasound was unremarkable. Computed tomography (CT) of the head without contrast was normal. Cerebrospinal fluid (CSF) analysis revealed normal cell counts (WBC $1 / \mu \mathrm{l}$ and $\mathrm{RBC} 1 / \mu \mathrm{l}$ ), raised glucose at
$6.7 \mu \mathrm{mol} / \mathrm{l}(2.2-3.9)$, and protein of $17 \mathrm{mg} / \mathrm{dl}(12-40)$. CSF Gram stain and cultures were negative.

He was treated with intravenous thiamine and began treatment with intravenous ceftriaxone, azithromycin, and doxycycline for community acquired pneumonia or possible rickettsial infection. A brain MRI done two days after admission showed hypointensity and slight oedema in the SCC on Tl weighted images (fig 1A), which did not enhance with contrast (fig 1D). There was markedly increased signal in this region on diffusion weighted imaging (DWI) (fig 1B). The lesion was slightly hyperintense on T2 weighted images (fig lC). Intracranial magnetic resonance angiography showed no abnormalities (data not shown). A transthoracic echocardiogram revealed normal left ventricular function with no evidence of masses or thrombi.

After admission the patient became more somnolent and inattentive. In this setting, detailed features of a callosal syndrome ${ }^{4}$ could not be demonstrated conclusively. He was intermittently oriented with prolonged response latency, and he had difficulty in following complex commands. He could not perform sequential tasks and had limb apraxias. Although naming and repetition were normal, his speech remained dysarthric. Glabellar, snout, and palmo-mental reflexes were present. Finger-nose testing was accurate bilaterally, but his gait was ataxic. Electroencephalography showed generalised rhythmic slowing without epileptiform discharges, consistent with mild encephalopathy. A sputum sample was positive by direct fluorescent antibody (DFA) for Legionella pneumophila, and Legionella pneumophila serogroup 1 antigens were present in the urine. In the light of these findings, specific testing to exclude coinfection with Mycoplasma was not undertaken. The antibiotic regimen was changed to intravenous ciprofloxacin with subsequent gradual improvement in mentation. A repeat brain MRI with contrast done 13 days after the initial study showed complete resolution of the SCC lesion (fig 1, panels E-H).

He was discharged one month later with normal blood glucose, liver function, and renal function. Despite mild residual dysarthria, he could follow complex commands with less left-right confusion. Although his gait ataxia persisted, he was able to move around independently with a walking frame.

\section{DISCUSSION}

Legionnaires' disease is commonly associated with confusion, dysarthria, and cerebellar signs of gait and limb ataxia. ${ }^{125}$ Our patient had cerebellar dysfunction and frontal release signs, indicating neurological dysfunction beyond areas of the brain which typically contribute fibres to the SCC: the temporo-parieto-occipital junction, the superior parietal lobule, and the occipital cortex. ${ }^{6}$ Despite frequent cerebellar signs in legionnaires' patients, CT and brain scintigraphy have not revealed cerebellar lesions in acute disease.

Occasionally, neuroimaging studies have shown abnormalities associated with various clinical syndromes in patients with neurological deficits and Legionella infections (table 1). ${ }^{12-13}$ Identified lesions vary from abscess $^{7}$ to 

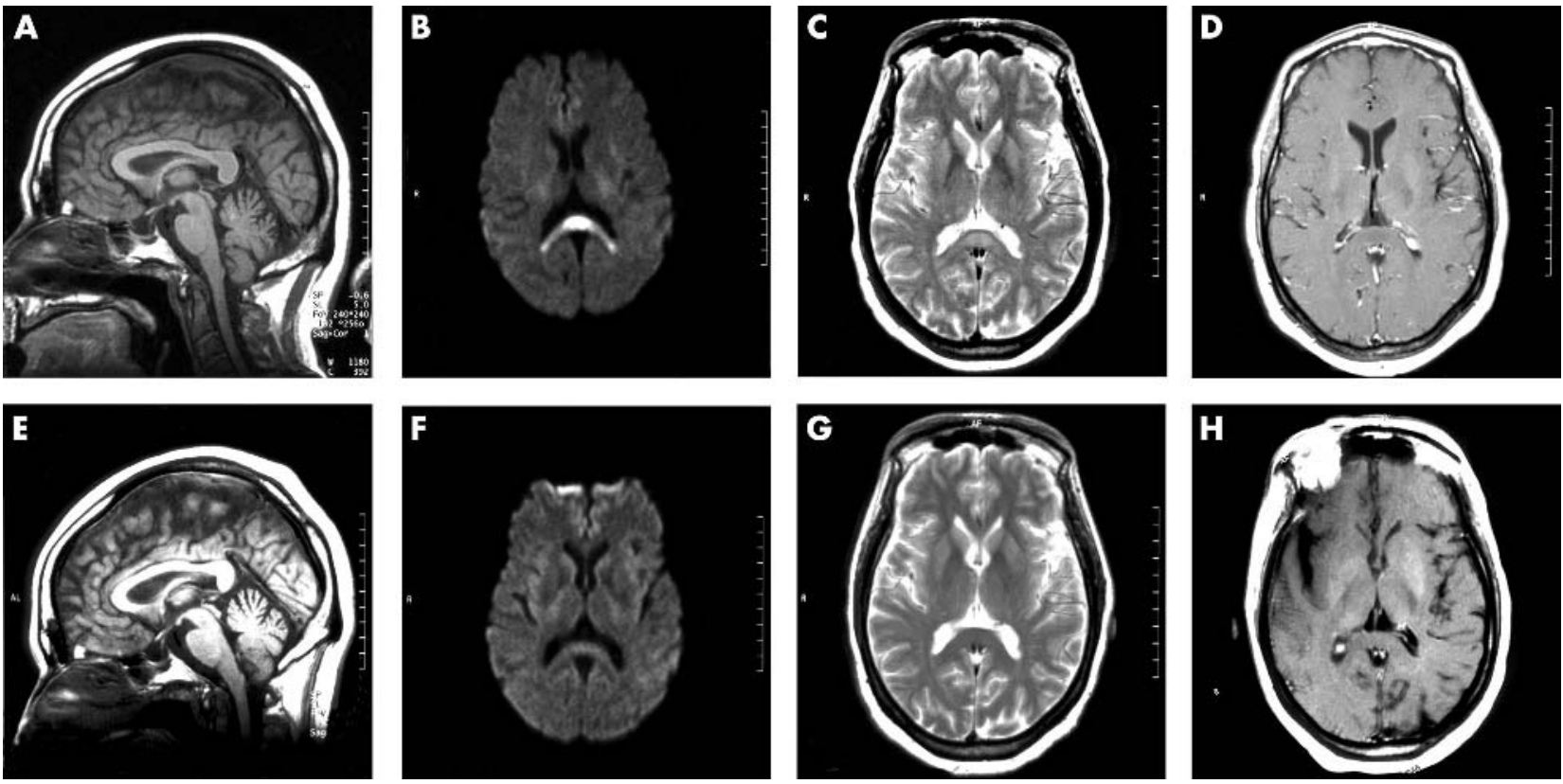

Figure 1 Brain magnetic resonance imaging (MRI) showing a callosal lesion on admission (A-D) and repeat brain MRI 13 days later (E-H). (A) Midsagittal T1 weighted image showing a hypointense lesion in the splenium of the corpus callosum. (B) Axial diffusion weighted imaging showing symmetrical hyperintensity in the same region. (C) Axial T2 weighted hyperintensity in the same region. (D) Postcontrast axial T1 weighted image showing hypointensity of the lesion without enhancement. (E-H) Corresponding images of the same patient 13 days later, with resolution of the callosal lesion.

Table 1 Brain imaging abnormalities in patients with neurological dysfunction and Legionella infections

\begin{tabular}{|c|c|c|c|}
\hline Reference & Age/sex & Clinical symptoms, signs, outcome & Imaging results \\
\hline Weir $(1982)^{1}$ & $53 / \mathrm{F}$ & $\begin{array}{l}\text { Headache, confusion, nuchal rigidity, dysarthria, extensor left toe, } \\
\text { ataxia of lower extremities; } 3 \text { years later she had ataxia in } \\
\text { her lower extremities more than in her upper extremities }\end{array}$ & $\begin{array}{l}\text { Head CT, normal in acute setting; head CT } \\
3 \text { years later, cerebellar atrophy }\end{array}$ \\
\hline Andersen $(1987)^{7}$ & $33 / M$ & $\begin{array}{l}\text { Headache, seizure, confusion, expressive aphasia, right sided } \\
\text { hyperreflexia, dysmetria in right upper extremity; Legionella } \\
\text { jordanis serum antibodies present at high titres; "normal" } \\
7 \text { months later except for "slightly impaired memory" }\end{array}$ & $\begin{array}{l}\text { Head CT, left temporoparietal abscess, } \\
\text { autologous leucocyte scan with } \\
\text { enhancement in same region }\end{array}$ \\
\hline Potasman $(1990)^{8}$ & $26 / F$ & $\begin{array}{l}\text { Fevers, headache, generalised seizure followed by confusion, left } \\
\text { hemiparesis, additional generalised seizures; Legionella bozemanii } \\
\text { antibodies in CSF; discharged } 1 \text { month later with normal } \\
\text { neurological examination }\end{array}$ & Head CT, effacement of sulci \\
\hline Johnson $(1984)^{2}$ & $\begin{array}{l}\text { Various, } 7 \text { patients } \\
\text { with imaging ( } 2 \\
\text { abnormal) }\end{array}$ & $\begin{array}{l}\text { Patient with cerebral oedema on head CT had confusion leading } \\
\text { to coma with otherwise normal neurological examination; patient } \\
\text { survived. Patient with multifocal lesions on brain scan presented in } \\
\text { coma and then developed left facial weakness, left hemiparesis, } \\
\text { hyperreflexia, and an extensor left toe by day } 12 \text { of hospital stay; } \\
\text { patient died and had necrotising haemorrhagic leucoencephalitis } \\
\text { with bacilli seen on Dieterle stain at necropsy }\end{array}$ & $\begin{array}{l}\text { Head CT, normal in } 4 \text { patients; brain } \\
\text { scintigraphy, normal in } 1 \text { patient; head } \\
\text { CT, cerebral oedema in one patient; brain } \\
\text { scintigraphy, multifocal lesions in one } \\
\text { patient }\end{array}$ \\
\hline Karim $(2002)^{10}$ & $21 / M$ & $\begin{array}{l}\text { Unresponsive to verbal commands, with nuchal rigidity on } \\
\text { admission, seizures followed, negative herpes simplex virus PCR; } \\
\text { Legionella pneumophila by DFA after bronchoalveolar lavage; "the } \\
\text { patient's neurologic condition gradually improved" }\end{array}$ & $\begin{array}{l}\text { Head CT, normal initially, slight } \\
\text { leptomeningeal enhancement on repeated } \\
\text { study; brain MRI, bilateral mesiotemporal } \\
\text { FLAIR hyperintensities }\end{array}$ \\
\hline Sommer $(2000)^{12}$ & $58 / M$ & $\begin{array}{l}\text { Three weeks after a probable Legionella pneumophila illness (non- } \\
\text { productive cough, fever, diarrhoea, abdominal pain, headache) the } \\
\text { patient developed headache, nausea, dizziness, left-beating } \\
\text { nystagmus and bilateral horizontal diplopia, lost consciousness; } \\
\text { complete recovery } 4 \text { weeks later with steroid treatment }\end{array}$ & $\begin{array}{l}\text { Brain MRI, confluent hyperintensity of the } \\
\text { bilateral periventricular and subcortical } \\
\text { white matter and left cerebellar peduncle } \\
\text { (ADEM) }\end{array}$ \\
\hline Spieker $(1998)^{11}$ & $35 / F$ & $\begin{array}{l}\text { Six weeks after Legionella cincinnatiensis CNS infection (confirmed } \\
\text { by PCR detection) the patient developed headache, agitation, } \\
\text { hallucinations, paranoia, generalised seizures, mutism, somnolence, } \\
\text { right facial dyskinesias, rigidity and dystonia; amnesia for } 2 \text { months, } \\
\text { other abnormalities on the neurological examination normalised }\end{array}$ & $\begin{array}{l}\text { Brain MRI, bilateral symmetrical } \\
\text { hyperintensity of the basal ganglia and left } \\
\text { subcortical white matter (ADEM); } \\
\text { improved on repeat imaging }\end{array}$ \\
\hline Platzeck $(1990)^{9}$ & $44 / M$ & $\begin{array}{l}\text { Legionella bozemanii pneumonia followed by tetraparesis and } \\
\text { severe midbrain syndrome; almost complete neurological recovery } \\
\text { after several weeks }\end{array}$ & $\begin{array}{l}\text { Head CT, normal; brain MRI, symmetrical, } \\
\text { bilateral foci of demyelination in the brain } \\
\text { stem }\end{array}$ \\
\hline Easterbrook $(1992)^{13}$ & 19/M & $\begin{array}{l}\text { Patient presented in coma with fever, CSF leucocytosis, Legionella } \\
\text { pneumophila and Mycoplasma pneumoniae coinfection documented } \\
\text { serologically; developed quadriplegia, anarthria, and dysphagia } \\
\text { with little improvement }\end{array}$ & $\begin{array}{l}\text { Head CT, normal on admission; brain } \\
\text { MRI, } 6 \text { weeks later, multifocal cerebral } \\
\text { white matter disease }\end{array}$ \\
\hline
\end{tabular}

ADEM, acute disseminated encephalomyelitis; CSF, cerebrospinal fluid; CT, computed tomography; FLAIR, fluid attenuated inversion recovery; MRI, magnetic resonance imaging; PCR, polymerase chain reaction. 
cerebral oedema ${ }^{28}$ on $\mathrm{CT}$, while brain scintigraphy revealed multifocal lesions in one patient. ${ }^{2}$ More recently, brain MRI studies in two patients have shown bilateral symmetrical foci of brain stem demyelination in Legionella bozemanii pneumonia ${ }^{9}$ and bilateral mesial temporal FLAIR MRI hyperintensities following seizures in acute legionnaires' disease. ${ }^{10}$ Two patients developed acute disseminated encephalomyelitis (ADEM) several weeks after infection with Legionella species. ${ }^{11}{ }^{12}$ Postinfectious encephalomyelitis has also occurred in a 19 year old man who had documented coinfection with Legionella pneumophila and Mycoplasma pneumoniae..$^{13}$

The frequent finding of cerebellar signs in legionnaires' patients without neuroimaging evidence of cerebellar lesions may have several potential explanations. CT and brain scintigraphy may not be sensitive enough for routine detection of cerebellar lesions in legionnaires' patients. Additionally, patients developing an acute or postinfectious cerebellar ataxia often have normal cerebellar MRI findings, while functional imaging with single photon emission tomography (SPECT) may show marked diffuse cerebellar perfusion abnormalities. ${ }^{14}$

Our patient's SCC lesion may have generated more widespread cortical or cerebellar dysfunction through diaschisis or other mechanisms. Widespread bilateral alterations in cerebral blood flow and metabolism on positron emission tomography (PET) can occur in patients with Marchiafava Bignami disease who have a clinical presentation and neuroimaging findings similar to our patient. ${ }^{15}$ Similarly, in a series of 13 cases of intractable epilepsy undergoing total callosotomy, five developed persistent dysarthria and gait difficulties, while those receiving anterior two thirds or subtotal callosotomy did not develop these problems. ${ }^{16}$ This implies that posterior corpus callosum damage may be an important cause of dysarthria and gait ataxia.

In addition to Marchiafava Bignami disease, several other conditions with reversible SCC lesions are associated with clinical presentations similar to our patient. High altitude cerebral oedema (HACE), for example, is often associated with confusion, ataxia, and reversible SCC lesions. ${ }^{17}$ Reversible SCC lesions also occur in haemolytic-uraemic syndrome, ${ }^{18}$ rotavirus infection, ${ }^{19}$ and acute cerebellitis. ${ }^{20}$

Hackett et al hypothesised ${ }^{17}$ that SCC lesions in HACE are related to vasogenic oedema because they respond to steroids and resolve slowly without significant residual clinical deficit. In haemolytic-uraemic syndrome, $E$ coli $\mathrm{O}-157$ verotoxin binding to cerebral vascular endothelium with subsequent microvascular angiopathy and perivascular oedema is proposed as a mechanism for a reversible SCC lesion. ${ }^{18} \mathrm{~A}$ reversible SCC lesion in a child with rotavirus infection was hypothesised to represent cytotoxic oedema, given that the lesion was hyperintense on DWI, though the pathogenic mechanism is unknown. ${ }^{19}$

Brain MRI in our patient showed DWI hyperintensity in the SCC that would customarily indicate cytotoxic oedema. Our patient did not receive corticosteroids, given the usual full neurological recovery in legionnaires' patients and the unproven benefit of corticosteroids in treating cytotoxic oedema. The resolution of his SCC lesion by MRI and the parallel clinical improvement is similar to the resolution of DWI changes in patients who develop acute metabolic decompensation and encephalopathy in maple syrup urine disease. ${ }^{21}$ When symptomatic, patients with this condition develop reversible regions of significantly restricted proton diffusion in large areas of white matter without evidence of volume loss or tissue damage. This may represent intramyelinic sheath oedema that reverses after metabolic correction. ${ }^{21}$ Perhaps resolution of intramyelinic sheath oedema explains the reversibility of our patient's SCC lesion and the absence of signs of infarction or necrosis.
The pathogenic mechanism of Legionella induced neurological dysfunction is unknown. In very rare instances, the Gram negative bacterium has been demonstrated directly in postmortem brain tissue ${ }^{2}$; however, this is usually not the case. ${ }^{3}$ An endotoxin effect accounting for the neurological dysfunction has been hypothesised, ${ }^{232}$ similar to the mechanism proposed for haemolytic-uraemic syndrome. ${ }^{18}$ As most patients with Legionella infections and CNS dysfunction have normal CSF leucocyte counts, with few exceptions,,$^{13}{ }^{23}$ an endotoxic effect of Legionella spp would be a plausible mechanism for CNS disease. This endotoxin hypothesis is supported by evidence that Legionella pneumophila produces a cytotoxin that kills Chinese hamster ovary cells. ${ }^{24}$ Legionella spp can also generate pores in eukaryotic cell membranes, leading to cell death by osmotic lysis. ${ }^{25}$ Such features of Legionella pneumophila may have been responsible for our patient's reversible SCC lesion, though the local vulnerability of the SCC remains unexplained.

Application of newer imaging methods such as MRI may show that brain lesions in legionnaires' disease are more common than previously appreciated. Functional imaging with SPECT and PET may also provide evidence of CNS dysfunction in legionnaires' patients who show neurological signs without structural lesions on MRI. Resolution of oedematous brain lesions may account for the paucity of findings with less sensitive neuroimaging studies (CT and brain scintigraphy), the rarity of neuropathological findings, and the customarily complete neurological recovery in legionnaires' patients. Legionnaires' disease-like haemolyticuraemic syndrome, rotavirus encephalopathy, and acute cerebellitis-should be included in the differential diagnosis of infectious conditions associated with reversible lesions of the corpus callosum.

\section{ACKNOWLEDGEMENTS}

We would like to thank Drs T P Bleck and G F Wooten for helpful criticism of our manuscript.

\section{Authors' affiliations \\ J C Morgan, Medical College of Georgia, Department of Neurology, Augusta, Georgia, USA \\ R Cavaliere, V C Juel, University of Virginia, Department of Neurology, Charlottesville, Virginia, USA \\ Competing interests: none declared}

Correspondence to: Dr Vern C Juel, PO Box 800394, Charlottesville, VA 22908-0394, USA; vi2n@virginia.edu

Received 28 March 2003

In revised form 19 August 2003

Accepted 24 August 2003

\section{REFERENCES}

1 Weir Al, Bone I, Kennedy DH. Neurological involvement in legionellosis. J Neurol Neurosurg Psychiatry 1982;45:603-8.

2 Johnson JD, Raff MJ, Van Arsdall JA. Neurologic manifestations of Legionnaires' disease. Medicine 1984;63:303-10.

3 Pendlebury WW, Perl DP, Winn WC, et al. Neuropathologic evaluation of 40 confirmed cases of Legionella pneumonia. Neurology 1983;33:1340-4.

4 Habib M. Corpus callosum disconnection syndromes and functional organization of the corpus callosum in adults. Neurochirurgie 1998;44(suppl 1):102-9.

5 Shetty KR, Cilyo CL, Starr BD, et al. Legionnaires' disease with profound cerebellar involvement. Arch Neurol 1980;37:379-80.

6 de Lacoste MC, Kirkpatrick JB, Ross ED. Topography of the human corpus callosum. J Neuropathol Exp Neurol 1985;44:578-91.

7 Andersen BB, Søgaard I. Legionnaires' disease and brain abscess. Neurology 1987:37:333-4.

8 Potasman I, Liberson A, Krimerman S. Legionella infection mimicking herpes encephalitis. Crit Care Med 1990; 18:453-4.

9 Platzeck C, Foerster EC, Schneider MU, et al. Encephalitis in Legionella bozemanii pneumonia. Dtsch Med Wochenschr 1990;115:1956-9.

10 Karim A, Ahmed S, Rossoff U. Legionnaire's disease associated with acute encephalitis and arrhythmia. Crit Care Med 2002;30:1028-9. 
11 Spieker S, Petersen D, Rolfs A, et al. Acute disseminated encephalomyelitis following Pontiac fever. Eur Neurol 1998;40:169-72.

12 Sommer JB, Erbguth FJ, Neundorfer B. Acute disseminated encephalomyelitis following Legionella pneumophila infection. Eur Neurol 2000;44:182-4.

13 Easterbrook PJ, Smyth EG. Post-infectious encephalomyelitis associated with Mycoplasma pneumoniae and Legionella pneumophila infection. Postgrad Med J 1992;68:124-8.

14 Daaboul Y, Vern BA, Blend MJ. Brain SPECT imaging and treatment with IVIg in acute post-infectious cerebellar ataxia: case report. Neurol Res 1998;20:85-8

15 Sano M, Ishii K, Suzuki T, et al. Reductions in the bilateral parietal and occipital cerebral blood flow and metabolism in a patient with MarchiafavaBignami disease. J Neurol 1999;246:607-8.

16 Cendes F, Ragazzo PC, da Costa V, et al. Corpus callosotomy in treatment of medically resistant epilepsy: preliminary results in a pediatric population. Epilepsia 1993;34:910-17

17 Hackett PH, Yarnell PR, Hill R, et al. High-altitude cerebral edema evaluated with magnetic resonance imaging. JAMA 1998;280:1920-5.
18 Ogura H, Takaoka M, Kishi M, et al. Reversible MR findings of hemolytic uremic syndrome with mild encephalopathy. Am J Neuroradiol 1998;19:1144-5.

19 Kobata R, Tsukahara H, Nakai A, et al. Transient MR signal changes in the splenium of the corpus callosum in rotavirus encephalopathy: value of diffusion-weighted imaging. J Comput Assist Tomogr 2002;26:825-8.

20 Kato Z, Kozawa R, Hashimoto K, et al. Transient lesion in the splenium of the corpus callosum in acute cerebellitis. J Child Neurol 2003;18:291-2.

21 Jan W, Zimmerman RA, Wang Z, et al. MR diffusion imaging and MR spectroscopy of maple syrup urine disease during acute metabolic decompensation. Neuroradiology 2003;45:393-9.

22 Wong KH, Moss CW, Hochstein DH, et al. "Endotoxicity" of the Legionnaires' disease bacterium. Ann Intern Med 1979:90:624-7.

23 Harris L. Legionnaires' disease associated with acute encephalomyelitis. Arch Neurol 1981;38:462-3.

24 Friedman RL, Iglewski BH, Miller RD. Identification of a cytotoxin produced by Legionella pneumophila. Infect Immun 1980;29:271-4.

25 Kirby JE, Vogel JP, Andrews HL, et al. Evidence for pore-forming ability by Legionella pneumophila. Mol Microbiol 1998;27:323-36. 